\title{
Vibrational excitation of methane by slow electrons revisited: theoretical and experimental study
}

\author{
R Čurík ${ }^{1}$, P Čársky ${ }^{1}$ and M Allan ${ }^{2}$ \\ ${ }^{1}$ J Heyrovský Institute of Physical Chemistry, Academy of Sciences of the Czech Republic, \\ 18223 Prague 8, Czech Republic \\ ${ }^{2}$ Department of Chemistry, University of Fribourg, CH-1700 Fribourg, Switzerland \\ E-mail: roman.curik@jh-inst.cas.cz
}

\begin{abstract}
We have calculated and measured differential and integral cross sections for vibrationally inelastic scattering of electrons by methane molecules. The calculations were carried out using the discrete momentum representation (DMR) method. We solved the two-channel Lippmann-Schwinger equation in the momentum space. The interaction between the scattered electron and the target molecule is described by the exact static-exchange potential. Correlation-polarization forces were included by a simple local density functional theory potential of Perdew and Zunger (1981 Phys. Rev. B 23 5048). The cross sections calculated in this way agree very well with our measurement and with other more recent experimental data, but are larger than some older experimental and theoretical results.
\end{abstract}

\section{Introduction}

Knowledge of electron impact vibrational excitations of methane is important in a wide variety of technological and atmospheric applications. Methane has been identified as a source of significant infrared absorption in the atmospheres of Jupiter, Saturn, Uranus and Neptune (Broadfoot et al 1986). Moreover, the methane molecule is involved in the network of greenhouse gas kinetics of Earth's atmosphere. Other applications are found in gaseous discharges, gaseous laser media, radiation detectors and in electron-impact-induced chemical reactions on surfaces.

As a result of the interests from these various fields, there has been considerable amount of experimental and theoretical work devoted to the study of electron-methane collisions in the last two decades. While calculation and measurement of elastic scattering cross sections is a well-established task (for a review, see Bundschu et al 1997), attempts at a theoretical analysis of vibrational excitation cross sections are rather rare.

Both off-shell and adiabatic techniques were applied for a single-centre expansion method by Althorpe et al (1995) and for a complex Kohn variational method by Rescigno et al (1995). Excitations of the symmetric mode have been analysed for several $\mathrm{T}_{d}$ molecules by Cascella et al (2001a) using local exchange and polarization with adiabatic approximation for the motion of the nuclei. Comparison between local and separable exchange models and their impact on the adiabatic excitations of all vibrational modes for methane can be found in Cascella et al (2001b). The latest calculations by Nishimura and Gianturco (2002) used a more rigorous treatment of nuclear dynamics via a close-coupling formalism, although exchange and correlation-polarization effects were included in the local approximation.

In the experimental field it is important to note that several measurements of the cross sections for vibrational excitation were made and they will be listed in detail in section 4.

In this work, we therefore aim to carry out fully ab initio calculations for the vibrational excitations of the methane molecule with exact static-exchange contributions and a closecoupling scheme for the vibrational motion. Correlationpolarization forces are included by a density functional theory (DFT) potential introduced by Perdew and Zunger (1981). The theoretical results are evaluated by comparison with both existing experimental data and also with our new measurements, which extend the energy and angular ranges of the existing experimental cross sections. 


\section{Experiment}

The measurements of the vibrationally inelastic cross sections were performed using a spectrometer with hemispherical energy analysers (Allan 1992, 2005b, 2007). It is equipped with a magnetic angle changer (Read and Channing 1996, Zubek 1996), which permits measurements over the entire angular range $0^{\circ}-180^{\circ}$. The instrumental response function was determined on elastic scattering in helium and all spectra were corrected as described earlier (Allan 2005b). Absolute values of the cross sections were determined by the relative flow technique using the theoretical helium elastic cross sections of Nesbet (1979) as a reference. The confidence limit of the absolute cross section values is about $\pm 25 \%$. The cross sections have a narrow peak in the forward direction which is particularly difficult to measure, and whose measurement is affected by the angular resolution of the instrument, about $3^{\circ}$. The measurement is consequently less precise within the first $\sim 5^{\circ}$

\section{Theory}

\subsection{Overview of the DMR method}

The vibrationally inelastic DMR method was discussed in detail by Čurík and Čársky (2003) and only a brief summary is given here. We made use of the following approximations.

- We introduce a one-electron optical potential for the interaction between the scattered electron and the charge density of the molecule. Furthermore we retain only the first term of the optical potential expansion (see, for example, Joachain 1975), ending up with the staticexchange (SE) approximation.

- The SE approximation is corrected by a model DFT potential $V_{\mathrm{cp}}$ that accounts for orbital relaxation of the bound electrons and for the correlation between the scattered electron and the bound electrons. We used the interpolation formula suggested by Perdew and Zunger (1981).

- Nuclear dynamics is described by the rotationally frozen and vibrationally harmonic approximations. Moreover, for the vibrational space of each normal mode we use only a two-state approximation. Nishimura and Gianturco (2002) have shown that the contributions from the coupling with the higher vibrational states are negligible for the $\mathrm{CH}_{4}$ molecule in the whole energy regime from $0.5 \mathrm{eV}$ to $12 \mathrm{eV}$.

These approximations allow us to describe the scattering problem via the two-channel Lippmann-Schwinger equation in the three-dimensional momentum space:

$$
\begin{aligned}
& \left\langle\chi_{1} \boldsymbol{k}_{1}|\hat{T}| \chi_{0} \boldsymbol{k}_{0}\right\rangle=\left\langle\chi_{1} \boldsymbol{k}_{1}|\hat{U}| \chi_{0} \boldsymbol{k}_{0}\right\rangle \\
& \quad+\sum_{i=0}^{1} \int \mathrm{d} \boldsymbol{k} \frac{\left\langle\chi_{1} \boldsymbol{k}_{1}|\hat{U}| \chi_{i} \boldsymbol{k}\right\rangle\left\langle\chi_{i} \boldsymbol{k}|\hat{T}| \chi_{0} \boldsymbol{k}_{0}\right\rangle}{k_{0}^{2}-2 E_{i}-k^{2}+\mathrm{i} \varepsilon},
\end{aligned}
$$

where $\hat{U}$ stands for twice the interaction potential $\hat{V}, E_{0}=0$ (for the elastic channel) is the energy of the vibrational ground state and $E_{1}$ is the energy of the first excited vibrational state.
These two states are described by the vibrational functions $\chi_{0}$ and $\chi_{1}$, respectively. The vectors $\boldsymbol{k}_{0}$ and $\boldsymbol{k}_{1}$ represent the plane-wave functions for the incoming and outgoing electrons, respectively.

Numerical discretization ( $p$ and $i$ run through abscissas of the radial and angular quadratures, respectively)

$$
\int \mathrm{d} k \int \mathrm{d} \hat{\boldsymbol{k}} g(\boldsymbol{k}) \rightarrow \sum_{p}^{N \mathrm{RAD}} w_{p} \sum_{i}^{N \text { ANG }} w_{i} g\left(\boldsymbol{k}_{p i}\right)
$$

of the integral on the rhs of equation (1) leads to a set of two-coupled matrix equations:

$$
\begin{gathered}
\left(\begin{array}{ll}
\mathbb{T}_{00} & \mathbb{T}_{01} \\
\mathbb{T}_{10} & \mathbb{T}_{11}
\end{array}\right)=\left(\begin{array}{ll}
\mathbb{U}_{00} & \mathbb{U}_{01} \\
\mathbb{U}_{10} & \mathbb{U}_{11}
\end{array}\right)+\left(\begin{array}{cc}
\mathbb{U}_{00} & \mathbb{U}_{01} \\
\mathbb{U}_{10} & \mathbb{U}_{11}
\end{array}\right) \cdot\left(\begin{array}{cc}
\mathbb{G}_{0} & 0 \\
0 & \mathbb{G}_{1}
\end{array}\right) \\
\cdot\left(\begin{array}{ll}
\mathbb{T}_{00} & \mathbb{T}_{01} \\
\mathbb{T}_{10} & \mathbb{T}_{11}
\end{array}\right),
\end{gathered}
$$

with the interaction matrix elements defined as follows:

$$
\begin{gathered}
{\left[\mathbb{U}_{00}\right]_{p i, q j}=\left\langle\chi_{0} \boldsymbol{k}_{p i}|\hat{U}| \chi_{0} \boldsymbol{k}_{q j}\right\rangle} \\
{\left[\mathbb{U}_{11}\right]_{p i, q j}=\left\langle\chi_{1} \boldsymbol{k}_{p i}|\hat{U}| \chi_{1} \boldsymbol{k}_{q j}\right\rangle} \\
{\left[\mathbb{U}_{01}\right]_{p i, q j}=\left\langle\chi_{0} \boldsymbol{k}_{p i}|\hat{U}| \chi_{1} \boldsymbol{k}_{q j}\right\rangle=\left[\mathbb{U}_{10}\right]_{p i, q j} .}
\end{gathered}
$$

The body-fixed scattering amplitudes for the vibrational transitions are obtained by the matrix inversion in equation (3). Evaluation of the matrix elements (4) was given by Čurík and Čársky (2003). More details about the quadrature scheme (2) of the singular kernels on the rhs of equation (1) can be found in Polášek et al (2000) and Čársky and Čurík (2006).

\subsection{Correlation-polarization potential}

Several models for a potential that includes both correlation and polarization effects in electron-molecule scattering problems were proposed by Perdew and Zunger (1981), O'Connell and Lane (1983) and Padial and Norcross (1984). Since then many authors have successfully utilized a simple and local form of the correlation energy provided by these electron-gas type simulations noted above. Among these we mention applications to the elastic electron-molecule collisions (Čurík et al 2000) and vibrationally and rotationally inelastic processes (Cascella et al 2001a, Telega et al 2004). In contrast to most of the DFT potentials used in quantum chemistry these models do not contain exchange interaction and they are based on a hybridization of the local electrongas theory for short distances and the asymptotic form of the polarization potential as

$$
V_{\mathrm{cp}}=\left\{\begin{array}{ll}
V_{\mathrm{c}}, & r \leqslant r_{0} \\
V_{\mathrm{p}}=-\frac{\alpha_{0}}{2 r^{4}}, & r>r_{0}
\end{array},\right.
$$

where the dipole polarization potential $V_{\mathrm{p}}$ is spherically symmetric in the case of methane molecule. In the above equation $r_{0}$ is a matching radius where $V_{\mathrm{c}}=V_{\mathrm{p}}$ and $\alpha_{0}$ is the static isotropic polarizability of the molecule. The $V_{\text {cp }}$ potential (5) is energy independent and very simple to apply, depending only on the molecular charge density and polarizabilities. For its short-range part $V_{\mathrm{c}}$ we followed the 

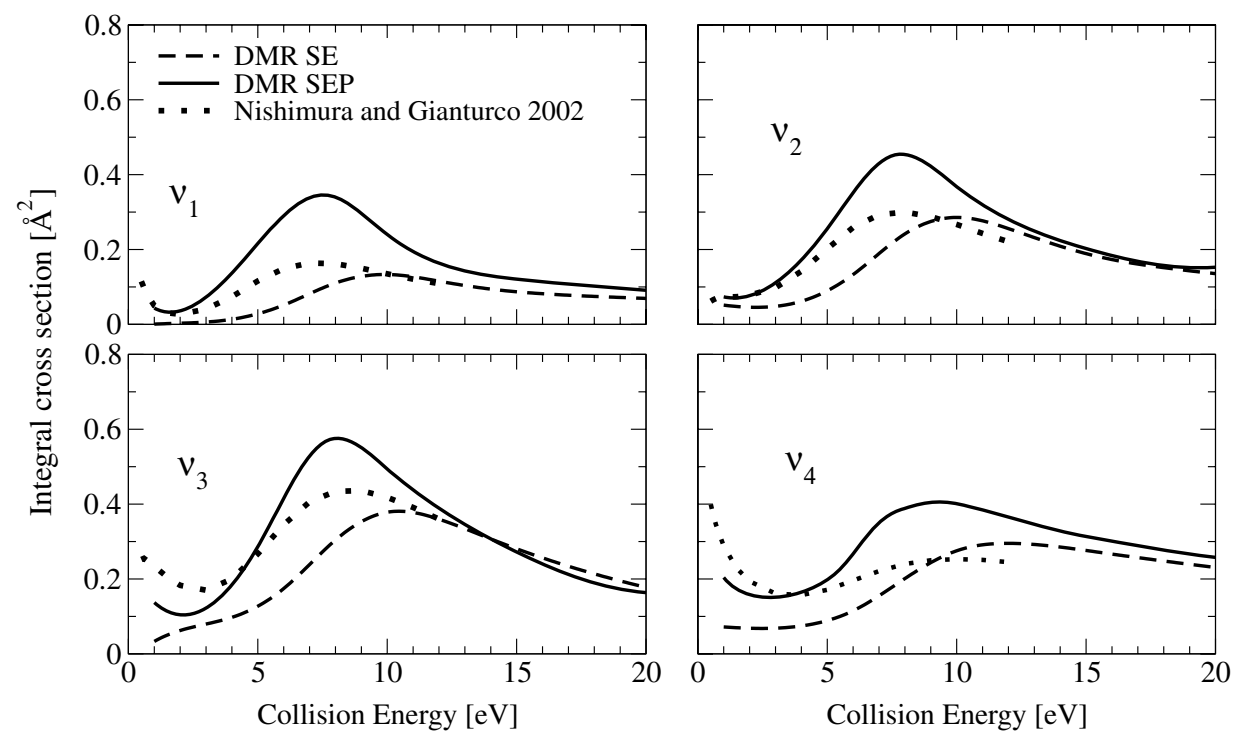

Figure 1. Rotationally summed $v=0 \rightarrow 1$ integral cross sections for the excitation of the four vibrational modes indicated. The broken curves represent our results with the SE optical potential while the full curves also include the correlation-polarization model (SEP), summarized in equation (5). We compare our results with the calculations of Nishimura and Gianturco (2002), displayed with dotted lines.

conclusions of Padial and Norcross (1984) by choosing the form of Perdew and Zunger (1981):

$V_{\mathrm{c}}(\vec{r})= \begin{cases}0.0311 \ln r_{\mathrm{s}}-0.0584, & \\ +0.00133 r_{\mathrm{s}} \ln r_{\mathrm{s}}-0.0084 r_{\mathrm{s}} & r_{\mathrm{s}}<1 \\ \frac{\gamma\left(1+\frac{7}{6} \beta_{1} \sqrt{r_{\mathrm{s}}}+\frac{4}{3} \beta_{2} r_{\mathrm{s}}\right)}{\left(1+\beta_{1} \sqrt{r_{\mathrm{s}}}+\beta_{2} r_{\mathrm{s}}\right)^{2}}, & r_{\mathrm{s}} \geqslant 1\end{cases}$

where the constants are $\gamma=-0.1423, \beta_{1}=1.0529, \beta_{2}=$ 0.3334 and the radius of a unity charge $r_{\mathrm{s}}$ is a function of the bound-electron density $\varrho(\vec{r})$

$$
r_{\mathrm{s}}=\left[\frac{3}{4 \pi \varrho(\vec{r})}\right]^{1 / 3}
$$

In order to calculate the matrix elements $\mathbb{U}_{01}$ in equation (4) we also need to evaluate derivatives of $V_{\mathrm{cp}}$ with respect to nuclear coordinates. This leads to the necessity of knowing the derivatives of the dipole polarizabilities in equation (5) and through equations (6) and (7) to derivatives of the electron density $\varrho(\vec{r})$. Both derivatives are evaluated by the use of standard quantum chemistry software as will be described below.

\subsection{Dipole polarizabilities}

Isotropic dipole polarizability of $\mathrm{CH}_{4}$ molecule has been a subject of numerous computational (Amos 1979, Wong et al 1991, Maroulis 1994) and experimental (Werner et al 1976, Hohm and Kerl 1993) studies. However the derivatives of the dipole polarizabilities are difficult to find in the literature. Therefore the first step in our computations was to determine all the independent components of polarizability tensor derivatives. We employed the linear response approach (Dalskov and Sauer 1998) in the following ab initio methods: second-order polarization propagator (SOPPA), multiconfiguration self-consistent field (MCSCF), coupled
Table 1. Dipole polarizability and its non-zero derivatives calculated by several ab initio methods with the daug-cc-pVTZ basis set. Atomic units are used throughout the table.

\begin{tabular}{llccc}
\hline Method & $\alpha_{0}$ & $\frac{\partial \alpha_{x x}}{\partial x_{H}}$ & $\frac{\partial \alpha_{y y}}{\partial x_{H}}$ & $\frac{\partial \alpha_{z z}}{\partial x_{H}}$ \\
\hline MCSCF & 16.15 & 2.81 & 1.85 & 1.85 \\
SOPPPA & 16.27 & 2.86 & 1.92 & 1.92 \\
CCSD & 16.41 & 2.92 & 1.92 & 1.92 \\
\hline
\end{tabular}

cluster singles and doubles (CCSD), with basis sets starting at aug-cc-pVDZ with the results being saturated at daug-ccpVTZ (Dunning 1989, Woon and Dunning 1994) or Sadlej's basis sets (Sadlej 1988). The results for the daug-cc-pVTZ basis are summarized in table 1. Our CCSD results give polarizability derivative for the symmetric stretch $R_{\mathrm{S}}$ of the $\mathrm{C}-\mathrm{H}$ bond $\partial \alpha_{0} / \partial R_{\mathrm{S}}=15.6$ a.u. to be compared with 15.7 a.u. calculated by Maroulis (1994). Furthermore the CCSD Born-Oppenheimer polarizability $\alpha_{0}=16.41$ a.u. needs to be corrected by an additional value of 0.88 a.u. that accounts for zero-point vibrations (Wong et al 1991). This correction pushes our calculated $\alpha_{0}$ to 17.28 a.u. to be compared with the experimental value of 17.26 a.u. of Hohm and Kerl (1993). The encouraging agreement between computed polarizabilities and available experimental and theoretical data allowed us to use the CCSD values of table 1 for the following scattering calculations.

\section{Results}

Figure 1 shows the calculated dependence of the integral inelastic $(0 \rightarrow 1)$ cross section on collision energy for all four vibrational modes. As can be seen the cross section is dominated by a broad shape resonance centred around $7.5 \mathrm{eV}$. The effect of the $V_{\mathrm{cp}}$ interaction defined in equation (5) appears to be negligible for the collision energies 

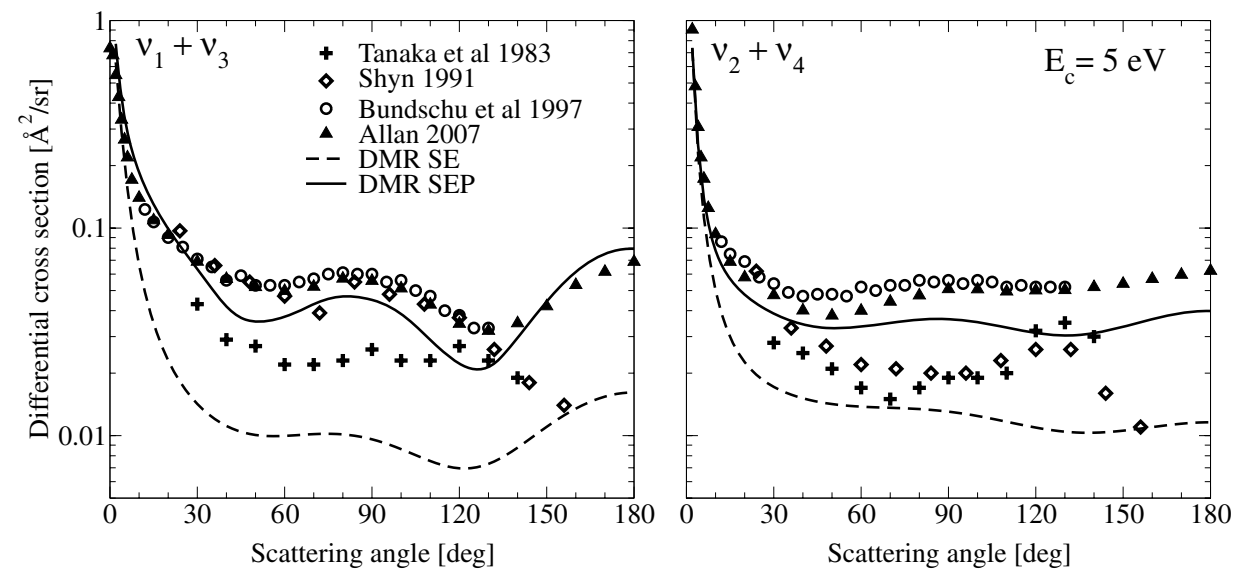

Figure 2. Rotationally summed $0 \rightarrow 1$ differential cross section for the collision energy of $5 \mathrm{eV}$. Broken curves represent our results with the SE optical potential while the solid lines also include the correlation-polarization given by equation (5) (SEP). The results of Tanaka et al (1983), Shyn et al (1991), Bundschu et al (1997) and Allan (2007) are shown for comparison. The left panel is for the composite stretching mode $v_{1}+v_{3}$ and the right panel displays data for the composite deformation mode $v_{2}+v_{4}$.
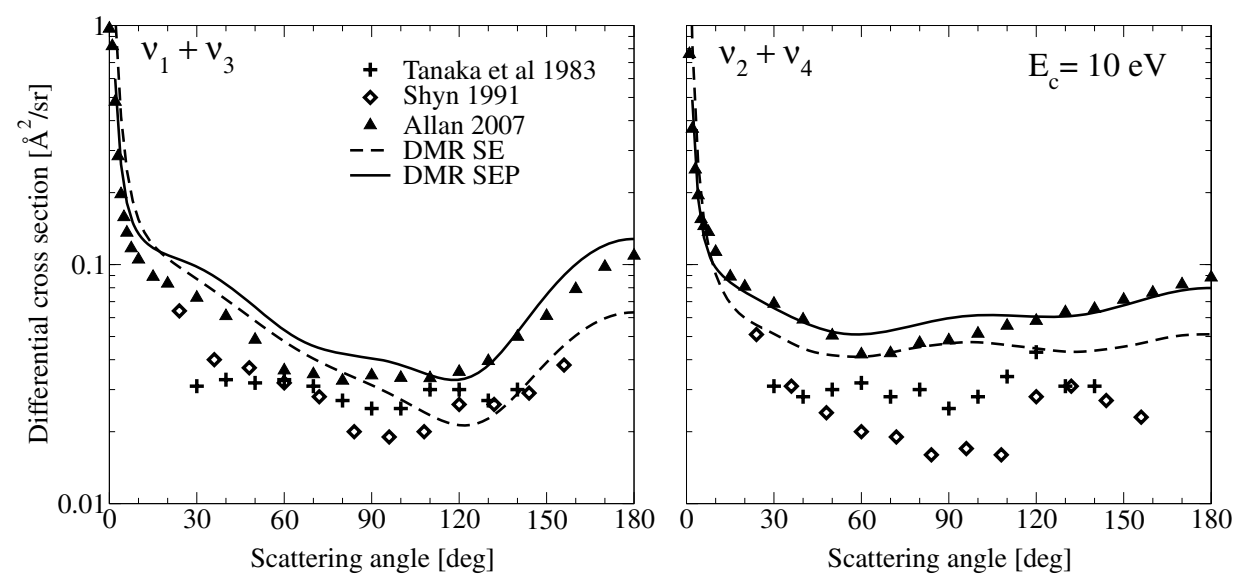

Figure 3. Rotationally summed $0 \rightarrow 1$ differential cross sections for the collision energy of $10 \mathrm{eV}$. Broken curves represent our results with the SE optical potential while the solid lines also include the SEP correlation-polarization model of equation (5). The results of Tanaka et al (1983), Shyn (1991) and Allan (2007) are shown for comparison. The left panel is for the composite stretching mode $v_{1}+v_{3}$ and the right panel displays data for the composite deformation mode $v_{2}+v_{4}$.

above $12 \mathrm{eV}$, but it shifts the shape resonance to lower energies and strongly enhances the cross section in the resonant regime. For a comparison figure 1 also displays results taken from Nishimura and Gianturco (2002). Their calculation treated nuclear dynamics in a close-coupling scheme as has been done in the present work. However, the main source of differences comes from the optical potential. The exchange part of the interaction was treated in a local semiclassical approximation in their work (Gianturco and Scialla 1987), while it is exact in the present study. Also, the previous calculations used a different correlation interaction $V_{\mathrm{c}}$, based on the electronelectron correlation energy as suggested by Carr et al (1961) and Carr and Maradudin (1964). Since the magnitudes of the cross sections of Nishimura and Gianturco (2002) are somewhere between our uncorrelated and correlated results, we conclude that either their correlation interaction or their exchange interaction (or both) are weaker than those of our model.
We further evaluate the two theoretical approaches by comparing our calculated differential cross sections with available experimental data at $5 \mathrm{eV}$ (in figure 2) and $10 \mathrm{eV}$ (in figure 3). The present experimental data are listed for selected angles in tables 2 and 3 . The two $\mathrm{C}-\mathrm{H}$ stretch vibrations $v_{1}$ and $v_{3}$, and also the two deformation vibrations $v_{2}$ and $v_{4}$ cannot be entirely resolved experimentally, even with high instrumental resolution, because of the overlap of the rotational band envelopes (Müller et al 1985, Allan 2005a). Only the sums of the differential cross sections can meaningfully be compared with the results of calculations. Each unresolved pair contains one infrared active mode (non-zero transition dipole moment) and the angular dependence of both cross sections is therefore peaked around the forward direction, both at $5 \mathrm{eV}$ and $10 \mathrm{eV}$ (figures 2 and 3), as expected (Itikawa 2000). The more recent measurements, those of Bundschu et al (1997) and Allan (2007), are in an excellent agreement at $5 \mathrm{eV}$, while the older experiments carried out by Tanaka et al (1983) are 
Table 2. Experimental DCS for vibrational excitation, measured as a function of scattering angle $\theta$, at $5 \mathrm{eV}$. The units are $10^{-22} \mathrm{~m}^{2} \mathrm{sr}^{-1}$.

\begin{tabular}{llllllllll}
\hline$\theta$ & 0 & 1 & 2 & 3 & 4 & 5 & 6 & 7.5 & 10 \\
$v_{2}+v_{4}$ & 251 & 183 & 90.5 & 48.2 & 30.7 & 21.9 & 17.3 & 12.5 & 9.35 \\
$v_{1}+v_{3}$ & 73.5 & 68.0 & 54.6 & 42.8 & 33.3 & 26.7 & 21.9 & 17.1 & 14.0 \\
$\theta$ & 15 & 20 & 30 & 40 & 50 & 60 & 70 & 80 & 90 \\
$\nu_{2}+v_{4}$ & 6.88 & 5.79 & 4.75 & 4.01 & 3.79 & 4.00 & 4.42 & 4.74 & 5.08 \\
$v_{1}+v_{3}$ & 10.9 & 9.27 & 6.89 & 5.69 & 5.20 & 4.98 & 5.22 & 5.71 & 5.57 \\
$\theta$ & 100 & 110 & 120 & 130 & 140 & 150 & 160 & 170 & 180 \\
$v_{2}+v_{4}$ & 5.07 & 4.95 & 5.01 & 5.03 & 5.20 & 5.39 & 5.69 & 5.96 & 6.23 \\
$v_{1}+v_{3}$ & 5.12 & 4.28 & 3.46 & 3.20 & 3.48 & 4.20 & 5.32 & 6.16 & 6.88 \\
\hline
\end{tabular}

Table 3. Experimental DCS for vibrational excitation, measured as a function of scattering angle $\theta$, at $10 \mathrm{eV}$. The units are $10^{-22} \mathrm{~m}^{2} \mathrm{sr}^{-1}$.

\begin{tabular}{llllllllll}
\hline$\theta$ & 0 & 1 & 2 & 3 & 4 & 5 & 6 & 7.5 & 10 \\
$v_{2}+v_{4}$ & 234 & 76 & 37 & 25 & 19.5 & 15.5 & 14.5 & 13.7 & 11.3 \\
$v_{1}+v_{3}$ & 97 & 82 & 48 & 28.4 & 19.7 & 15.8 & 13.6 & 11.7 & 10.5 \\
$\theta$ & 15 & 20 & 30 & 40 & 50 & 60 & 70 & 80 & 90 \\
$v_{2}+v_{4}$ & 8.91 & 8.09 & 6.86 & 5.89 & 5.05 & 4.21 & 4.26 & 4.70 & 4.82 \\
$v_{1}+v_{3}$ & 8.89 & 8.33 & 7.27 & 6.09 & 4.85 & 3.61 & 3.48 & 3.27 & 3.44 \\
$\theta$ & 100 & 110 & 120 & 130 & 140 & 150 & 160 & 170 & 180 \\
$v_{2}+v_{4}$ & 5.14 & 5.55 & 5.82 & 6.32 & 6.53 & 7.13 & 7.63 & 8.26 & 8.83 \\
$v_{1}+v_{3}$ & 3.36 & 3.35 & 3.56 & 3.95 & 4.99 & 6.10 & 7.90 & 9.79 & 10.9 \\
\hline
\end{tabular}

significantly lower. Measurements of Shyn (1991) exhibit similar behaviour for the mode $v_{2}+v_{4}$. In the case of $v_{1}+v_{3}$ mode the experimental data of Shyn (1991) closely follow those of Bundschu et al (1997) and Allan (2007); however they fall below them for scattering angles above $140^{\circ}$.

Note that since the experimental differential cross sections of Tanaka et al (1983), shown in figure 2, and of Shyn (1991) are lower than the more recent results of Bundschu et al (1997) and Allan (2007), they yield the integral cross sections that agree well with the calculation of Nishimura and Gianturco (2002). The present calculated cross sections, larger because of the more elaborate treatment of the exchange interactions, agree well with the more recent experimental cross sections of Bundschu et al (1997) and Allan (2007). For the sake of clarity not all available experimental data are included in figures 2 and 3 , but a complete comparison was given by Allan (2007).

Our SEP calculations agree very well with the experiments of Bundschu et al (1997) and Allan (2007), both in terms of the shape and the absolute magnitude of the cross section. The agreement extends even to large scattering angles, where the data of Shyn (1991) drop, particularly at $5 \mathrm{eV}$. Figure 2 also displays the role of correlation-polarization forces in the resonant region. Although their impact is dramatic our calculated results still lie somewhat lower for the $v_{2}+v_{4}$ mode for large scattering angles. This seems to indicate some portion of short-range part of the correlation potential $V_{\mathrm{c}}$ is missing since the quality of the short-range interaction strongly affects larger scattering angles.

The remarkable improvement brought by the inclusion of correlation and polarization is displayed even more visibly in figure 4. It shows the dependence of the differential cross section (DCS) on the collision energy for the fixed scattering angle $\vartheta=90^{\circ}$. This scattering angle was chosen because the resonance has a strong $\mathrm{d}$-wave contribution resulting in a significant enhancement of the DCS for $90^{\circ}$ (visible in the left panel of figure 2). The present experimental data are listed for selected energies in table 4.

The excellent agreement of the present SEP calculation with the present experiment and the experiment of Bundschu et al (1997) confirms that the present method of including correlation and polarization is successful. It works well over the entire resonant region and down to about $1 \mathrm{eV}$. The dramatic effect played by correlation and polarization at energies below about $12 \mathrm{eV}$ is evident, particularly for the symmetric stretch vibration $v_{1}$. As already stated above, the data of Tanaka et al (1983) and Shyn (1991) appear to be too low.

Finally, in order to complete our comparisons we also simulated electron energy-loss spectra (EELS). The sharp spectra represented by narrow vertical bars in figure 5 were spread with an energy width determined from the elastic peak of the measured spectra. This pragmatic approach bypasses the complications brought by the complex details of the rotational band envelopes (Müller et al 1985). As the last step we normalized the experimental spectra on the calculated elastic peak. The individual vibrations $v_{2}, v_{4}$ and $v_{1}, v_{3}$ are partially resolved in the energy-loss spectra and this form of comparison has thus the advantage of qualitatively indicating the degree of agreement for all four individual vibrations, particularly for the $v_{2}$ and $v_{4}$ pair. Again, figure 5 reveals an excellent agreement between the calculated and the measured cross sections for collision energies of $5 \mathrm{eV}$ and $20 \mathrm{eV}$. The calculation correctly reproduces the observation that $v_{2}$ is excited more strongly than $v_{4}$ at $5 \mathrm{eV}$ and vice versa at $20 \mathrm{eV}$.

\section{Summary and conclusions}

In the present calculations we used the discrete momentum representation to study the inelastic scattering of electrons 

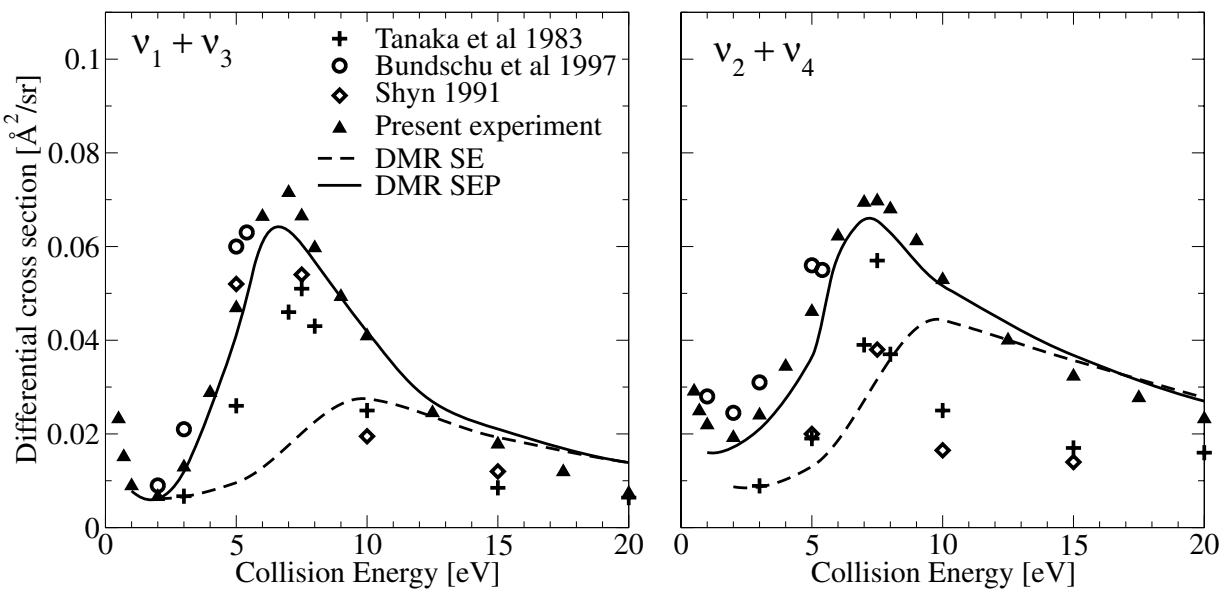

Figure 4. Rotationally summed $0 \rightarrow 1$ differential cross section as a function of the collision energy. The scattering angle is fixed at $\vartheta=90^{\circ}$. Our SE and SEP results are compared with the present experimental data and the previously measured cross sections of Tanaka et al (1983) and Bundschu et al (1997). The left panel is for the composite stretching mode $v_{1}+v_{3}$ and the right panel displays data for the composite deformation mode $v_{2}+v_{4}$.
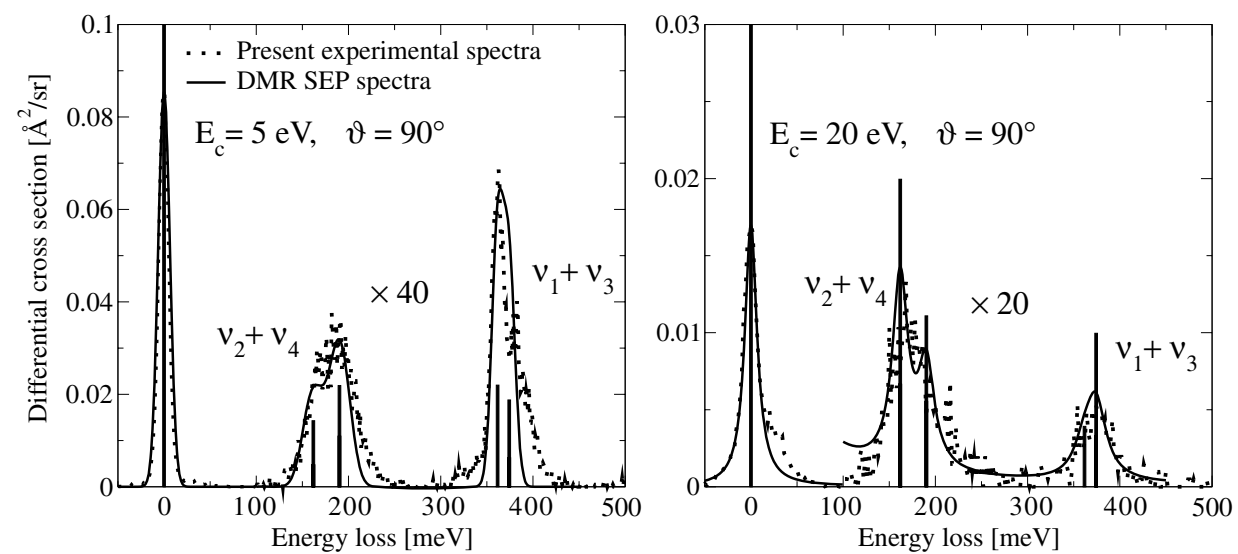

Figure 5. Computed and measured electron energy-loss spectra for the scattering angle $\vartheta=90^{\circ}$. The left panel is for the collision energy $E_{\mathrm{c}}=5 \mathrm{eV}$ and the right panel is for $E_{\mathrm{c}}=10 \mathrm{eV}$. Vertical bars represent the actual value of the differential cross section for the particular mode.

Table 4. Experimental DCS for vibrational excitation, measured as a function of the incident electron energy $E$, at $90^{\circ}$. The units are $10^{-22} \mathrm{~m}^{2} \mathrm{sr}^{-1}$.

\begin{tabular}{llllllllll}
\hline$E(\mathrm{eV})$ & 0.3 & 0.5 & 0.7 & 1.0 & 2.0 & 3.0 & 4.0 & 5.0 & 6.0 \\
$\nu_{2}+v_{4}$ & 3.42 & 2.91 & 2.49 & 2.19 & 1.92 & 2.40 & 3.44 & 4.62 & 6.22 \\
$v_{1}+v_{3}$ & - & 2.32 & 1.51 & 0.89 & 0.66 & 1.29 & 2.88 & 4.69 & 6.64 \\
$E(\mathrm{eV})$ & 7.0 & 7.5 & 8.0 & 9.0 & 10.0 & 12.5 & 15.0 & 17.5 & 20.0 \\
$\nu_{2}+v_{4}$ & 6.94 & 6.97 & 6.80 & 6.12 & 5.29 & 4.00 & 3.23 & 2.77 & 2.32 \\
$v_{1}+v_{3}$ & 7.15 & 6.65 & 5.97 & 4.93 & 4.09 & 2.45 & 1.78 & 1.19 & 7.55 \\
\hline
\end{tabular}

by the methane molecule. Our previously used (Čurík and Ćársky 2003) exact static-exchange potential was extended by the local DFT-based correlation-polarization contribution originally introduced by Perdew and Zunger (1981). Our calculation correctly reproduced the existence of the broad d-wave shape resonance which dominates the vibrationally inelastic collisions around $7.5 \mathrm{eV}$ and which was already known from the measurements of Tanaka et al (1983) and Shyn (1991) and which was found in the calculations by Nishimura and Gianturco (2002). Our present calculations predict that this resonance should be substantially more effective in enhancing vibrational excitation, however. We obtain larger cross sections for all vibrational modes, for the $v_{1}$ and $v_{4}$ modes by about a factor of 2 , in comparison with previous calculations. These larger values are in a very good accord with the experimental findings of Bundschu et al (1997), Allan (2007) and with the present experiment.

The current study represents our first attempt to improve the static-exchange inelastic matrix elements (4) by a local DFT correction derived from the derivatives of equations (5) 
and (6). From the good qualitative and quantitative agreements with the present experimental data and the measurements of Bundschu et al (1997) we conclude that such an optical potential may be successfully applied for vibrationally inelastic collisions with larger polyatomic molecules.

\section{Acknowledgments}

This work was supported by the Czech Ministry of Education (Kontakt ME857), the NSF (CHE-0446688), the project EIPAM sponsored by the European Science Foundation (grant no. PESC7-20), the Academy of Sciences of the Czech Republic (grant nos. A100400501, 1ET400400413 and KJB400400803), the Grant Agency of the Czech Republic (grant 202/08/0631) and the Swiss National Science Foundation (project no. 200020-113599/1). The calculations of polarizabilities were performed by Dr Ivana Paidarová (Heyrovský Institute, Prague) and Stephan Sauer (University of Copenhagen, Denmark). Their help is gratefully acknowledged.

\section{References}

Allan M 1992 J. Phys. B: At. Mol. Opt. Phys. 251559

Allan M 2005a J. Phys. B: At. Mol. Opt. Phys. 381679

Allan M 2005b J. Phys. B: At. Mol. Opt. Phys. 383655

Allan M 2007 Atomic and Molecular Data and Their Applications (AIP Conference Proceedings vol 91) ed E Roueff (New York: American Institute of Physics) p 107

Althorpe S C, Gianturco F A and Sanna N 1995 J. Phys. B: At. Mol. Opt. Phys. 284165

Amos R D 1979 Mol. Phys. 3833

Broadfoot A L et al 1986 Science 23374

Bundschu C T, Gibson J C, Gulley R J, Brunger M J, Buckman S J, Sanna N and Gianturco F A 1997 J. Phys. B: At. Mol. Opt. Phys. 302239

Čársky P and Čurík R 2006 Computational Chemistry: Reviews of Current Trends vol 10 ed J Leszczynski (Singapore: World Scientific)
Carr W J, Jr, Coldwell-Horsfall R A and Fein A E 1961 Phys. Rev. 124747

Carr W J, Jr and Maradudin A A 1964 Phys. Rev. A 133371

Cascella M, Curík R, Gianturco F A and Sanna N 2001a J. Chem. Phys. 114 1989-2000

Cascella M, Čurík R and Gianturco F A 2001b J. Phys. B: At. Mol. Opt. Phys. 34705

Čurík R, Gianturco F A and Sanna N 2000 J. Phys. B: At. Mol. Opt. Phys. 33615

Čurík R and Čársky P 2003 J. Phys. B: At. Mol. Opt. Phys. 362165

Dalskov E K and Sauer S P A 1998 J. Phys. Chem. A 1025269

Dunning T H, Jr 1989 J. Chem. Phys 901007

Gianturco F A and Scialla S 1987 J. Phys. B: At. Mol. Opt. Phys. 203171

Hohm U and Kerl K 1993 Mol. Phys. 80625

Itikawa Y 2000 Phys. Essays 13344

Joachain C J 1983 Quantum Collision Theory (Amsterdam: Elsevier) pp 577-621

Maroulis G 1994 Chem. Phys. Lett. 226420

Müller R, Jung K, Kochem K-H, Sohn W and Ehrhardt H 1985 J. Phys. B: At. Mol. Phys. 183971

Nesbet R K 1979 Phys. Rev. A 2058

Nishimura T and Gianturco F A 2002 J. Phys. B: At. Mol. Opt. Phys. 352873

O’Connell J and Lane N F 1983 Phys. Rev. A 271893

Padial N T and Norcross D W 1984 Phys. Rev. A 291742

Perdew J P and Zunger A 1981 Phys. Rev. B 235048

Polášek M, Juřek M, Ingr M, Čársky P and Horáček J 2000 Phys. Rev. A 61032701

Read F H and Channing J M 1996 Rev. Sci. Instrum. 672373

Rescigno T N, McCurdy C W, Orel A E and Lengsfield B H 1995 Computational Methods for Electron-Molecule Collisions ed W M Huo and F A Gianturco (New York: Plenum) pp 1-44 Sadlej A J 1988 Collect. Czech. Chem. Commun. 531995

Shyn T W 1991 J. Phys. B: At. Mol. Opt. Phys. 245169

Telega S, Bodo E and Gianturco F A 2004 Eur. Phys. J. D 29357

Tanaka H, Kubo M, Onodera N and Suziki A 1983 J. Phys. B: At. Mol. Opt. Phys. 162861

Werner H J and Meyer W 1976 Mol. Phys. 31855

Wong A T, Bacskay G B, Hush N S and Bogaard M P 1991 Mol. Phys. 741037

Woon D E and Dunning T H 1994 J. Chem. Phys. 1002975

Zubek M, Gulley N, King G C and Read F H 1996 J. Phys. B: At. Mol. Opt. Phys. 29 L239 